\title{
Incidencia de ETS y factores sociales en las trabajadoras sexuales en control sanitario en el Consultorio $\mathrm{N}^{\circ} 1$
}

\author{
SANDRA HERNÁNDEZ(1), IMAI NESVARA(2), CAROLINA TORRES(3), (†) CRISTIAN PEREDA(4) y ENRIQUE \\ HERNÁNDEZ(4)
}

\section{RESUMEN}

Las ETS son un problema de Salud Pública, debido a su alta incidencia, prevalencia, y morbimortalidad. El objetivo de este estudio es conocer la incidencia de ETS en las trabajadoras sexuales en control en el consultorio $N^{\circ} 1$ y su asociación con factores sociales como el Clima Social Preventivo (CSP). El presente es un estudio tipo descriptivo transversal, en el que se aplicó una encuesta a 120 trabajadoras sexuales, el que contenía preguntas abiertas y cerradas (comparadas con la información obtenida de la ficha clínica). Se estudiaron mediante regresión logística las variables incidencia de ETS, edad, escolaridad, nivel de ingreso, número de hijos, variables de tipo conductuales como edad inicio relaciones sexuales, número de parejas sexuales, uso preservativo, consumo alcohol-drogas, y variables del CSP estático (percepción del grupo de pares) y dinámico (lo que hace y recomienda el grupo de pares).

La incidencia de ETS en el grupo de estudio fue de 3,54\% por año. Se asoció significativamente con las siguientes variables: CSP estático malo (OR=1,34; 95\% IC: 1.13-3.2), CSP dinámico malo $(O R=4.80 ; 95 \%$ IC: $1.1-7.63)$, tienen hijos (OR=0.47; 95\% IC: $-0.35-0.78)$, vivir fuera de Santiago $(O R=1.27 ; 95 \%$ IC: $1.11-4.3)$, trabajar en la calle $(O R=3.92 ; 95 \%$ IC: $1.19-7.4)$, ingreso bajo $(O R=0.32 ; 95 \%$ IC: $-0.21-0.82)$, más de tres años de trabajo sexual $(O R=0.32 ; 95 \%$ IC: 0.02-1.19) y más de una pareja (OR=5.45; 95\% IC: 0.98-10.5).

La incidencia de ETS se asoció a un menor CSP estático y dinámico, menores ingresos, menos tiempo de trabajo sexual, más parejas sexuales. Un clima social preventivo favorable y otros factores sociales podrian influir en la conducta de las trabajadoras sexuales para reducir la incidencia de ETS, esta información contribuir al diseño de estrategias de prevención en salud pública.

Palabras clave: Factores sociales, estudios de prevalencia, ETS, SIDA, promiscuidad, factores de riesgo, factores protectores, análisis de regresión.

\footnotetext{
(1) Instituto de Epidemiología y Políticas de Salud Pública. Facultad de Medicina. Clínica Alemana Universidad del Desarrollo. hernandez.sa@gmail.com

(2) Estudiante de V año de Medicina. Universidad de Chile.

(3) Consultorio La Reina.

(4) Escuela de Salud Pública. Facultad de Medicina. Universidad de Chile.
} 


\section{ABSTRACT}

SEXUALLY TRANSMITTED DISEASES INCIDENCE AND SOCIAL FACTORS AMONG FEMALE SEX WORKERS UNDERGOING HEALTH FOLLOW-UP AT THE CONSULTORIO $\mathrm{N}^{\circ} 1$ AMBULATORY CLINIC.

In view of their high incidence, prevalence and morbimortality, STD are a Public Health issue. The purpose of the present study is to assess the incidence of STD among female sex workers undergoing health follow-up at the Consultorio $N^{\circ} 1$ ambulatory clinic and the association betwee STD and social factors such as the Preventive Social Atmosphere (PSA). The present work is a cross-sectional descriptive prevalence study, consisting of a survey responded by 120 female sex workers, which included open and closed questions (the answers were compared to the information on the clinical file). The STD variables: incidence, age, education, level of income, and number of children, the behavioral variables such as age at first intercourse, number of sexual partners, condom use, alcohol-drug intake, and both static PSA (peer group perception) and dynamic PSA (what the peer group does and recommends) variables were analyzed by logistic regression. The STD incidence in the study group was 3,54\% per year. The latter related significantly to the following variables: poor static PSA (OR=1.34; 95\% CI: 1.13-3.2), poor dynamic PSA (OR=4.80; 95\% CI: 1.1-7.63), having children (OR=0.47; 95\% CI: -0.35-0.78), living outside Santiago $(O R=1.27 ; 95 \%$ CI: 1.11-4.3), working on the street (OR=3.92; 95\% CI: 1.19-7.4), low income $(O R=0.32$; $95 \%$ CI: -0.21-0.82), more than three years of sex work $(O R=0.32 ; 95 \%$ CI: $0.02-$ 1.19) and more than one partner $(O R=5.45$; 95\% CI: 0.98-10.5).

The incidence of STD correlated with a poorer static and dynamic PSA, a lower income, shorter time as sex worker, more sex partners. A favorable preventive social atmosphere and other social factors might influence the behavior of sex workers to decrease the incidence of STD. The information herein discussed might contribute to the design of further public health prevention strategies.

Keywords: social factors, prevalence studies, STD, AIDS, promiscuity, risk factors, protective factors, regression analysis.

\section{INTRODUCCIÓN}

A pesar de los esfuerzos desplegados por organismos internacionales como la OPS, OMS, ONU, las ETS siguen siendo un problema de importancia para la Salud Pública mundial, debido a su alta prevalencia e incidencia ${ }^{1}$, elevados $\operatorname{costos}^{3}$ y elevada morbilidad y mortalidad $^{4,5}$. Según la OMS, 30 a 40 millones de adultos y niños estarían infectados con VIH al $2000^{1}$ y 333 millones de infectados por ETS ${ }^{2,3}$. Se ha determinado la existencia de vulnerabilidad sociocultural, ya que se afectan más los grupos marginales y con bajo acceso a la salud, también existe mas riesgo en los jóvenes $^{7,8}$, mujeres ${ }^{6,9}$ y en grupos de alto riesgo como trabajadoras sexuales y drogadictos endovenosos, estos últimos debido a su conducta sexual y consumo de alcohol y drogas 10,11 . En Chile las ETS son también un importante problema de Salud Pública, presentando una incidencia estimada de 71.6 casos por 100.000 habitantes ${ }^{12}$.

En numerosos estudios se demuestra que existe asociación entre conductas preventivas como el uso de preservativo ${ }^{13-17}$ y la abstinencia sexual ${ }^{18}$, en la reducción de la incidencia de ETS en trabajadoras sexuales y jóvenes.

La OMS ha propuesto para el control y prevención la reducción de las conductas de riesgo en la población mundial y en los grupos con conductas de riesgo ${ }^{6}$. Algunas de estas propuestas son la educación sexual de la 
población general ${ }^{19,20}$, el uso de preservativo, abstinencia y sexo seguro ${ }^{21-23}$.

Se sabe que factores sociales influirían en las conductas protectoras o de riesgo de grupos de riesgo como las trabajadoras sexuales. Uno de estos factores determinantes de estas conductas es el clima social.

El término "Clima Social" se origina del concepto de conciencia colectiva, es decir, "lo que el grupo siente, piensa y actúa es diferente de lo que piensa cada uno de los individuos que componen el grupo" 24 . Posteriormente nace el concepto de "Clima Social Preventivo", que se refiere a que cualquier medida de prevención adoptada frente a problemas de salud tiene menos o nulo efecto si los ambientes sociales continúan promoviendo el riesgo. Sin embargo, si las conductas de riesgo que se quieren prevenir son sancionadas socialmente por el grupo, se facilita el control

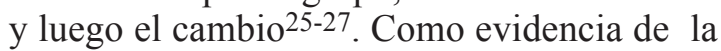
importancia del clima social en la conducta, datos de la literatura señalan que las conductas de riesgo individuales serían menos importantes en la adquisición de ETS que aquellas colectivas.

En Chile no existen estudios que hablen del CSP y menos aun que establezcan la asociación que existe con el comportamiento sexual y la incidencia de ETS. El propósito de este estudio es conocer la incidencia de ETS en un grupo de riesgo, las trabajadoras sexuales y su relación con algunos factores sociales como el clima social preventivo.

\section{MATERIAL Y MÉTODO}

Estudio descriptivo transversal. Se estimó una muestra representativa de 120 trabajadoras sexuales de un universo correspondiente a 1100 trabajadoras en control sanitario en el Centro de ETS del Consultorio $N^{\circ} 1$ de Santiago. Se utilizo la fórmula para calcular el $\mathrm{n}$ de poblaciones limitadas y se acordó trabajar con un intervalo de confianza de $95 \%$ un error $(\mathrm{d}=4 \%)$ y una probabilidad $\mathrm{P}=50 \%$. Se estimó una tasa de rechazo de $20 \%$ en la corrección del $n$ muestral. La técnica de muestreo realizada fue aleatorio simple, para ello cada día de la semana por un mes se sortearon los números de los pacientes citados ese día, encuestándose seis por día; cuando un paciente faltó se eligió el consecutivo en la lista.

La medición principal fue la incidencia declarada de ETS por persona en el último año, esta variable fue dicotomizada. Las variables independientes fueron: edad; estado conyugal; hijos; escolaridad; domicilio; lugar de trabajo; nivel de ingreso; edad de inicio de relaciones sexuales; tiempo de trabajo sexual; conducta sexual; número de parejas sexuales; comercio sexual; uso de preservativo; consumo de alcohol o drogas. El CSP de ETS fue definido como "lo que piensan, sienten, recomiendan y hace el grupo para prevenir las ETS. Existen dos tipos de CSP evaluados, con 2 categorías: bueno y malo. El CSP estático es la percepción de aceptación o rechazo por el grupo de pares sobre la prevención de ETS. El CSP dinámico fue definido como "lo que recomienda o sugiere el grupo de pares sobre prevención de ETS o si han decidido en grupo conductas preventivas en ETS". Las variables del estudio fueron categorizadas de acuerdo información obtenida de estudios anteriores, las categorías fueron definidas previas al análisis de los datos (Tabla 1) Las dimensiones y categorías del CSP fueron creadas de acuerdo a una metodología validada previa ${ }^{25-28}$.

Como instrumento se usó una encuesta que contó con preguntas abiertas y cerradas, para el CSP las preguntas estaban validadas por estudios anteriores ${ }^{26,27}$. La entrevista fue realizada por dos entrevistadores entrenados a todas las trabajadores sexuales que asistieron al control sanitario durante un mes, a quienes se les informó previamente el propósito del estudio, el carácter de confidencial y voluntario (consentimiento informado). Se realizo un piloto en el $10 \%$ de la muestra que permitió validar la encuesta durante la primera semana del trabajo en terreno. Un $10 \%$ de los datos se verificación con la ficha clínica y se creo un manual instructivo para los entrevistadores.

El análisis estadístico se procesó mediante STATA 5,0. Para el análisis de datos categóricos se uso tablas de estadísticas básicas (promedios máximos, mínimo, frecuencia), y tablas $2 \times 2$ para asociación (chi2, OR). E1 análisis 
multivariado de asociación se realizó mediante regresión logística (chi2, OR, p(z)). El Pvalue significativo fue $<0.05$.

\section{RESULTADOS}

El 28,33\% de las trabajadoras sexuales en control han tenido una o más ETS en el último año. La incidencia declarada de ETS fue de $3.54 \%$ episodios por persona-año, mientras que la incidencia por persona fue de 3,04\% al año, un porcentaje menor declaro 2 ETS en el año. No hubo episodios declarados de VIH-SIDA. Las patologías más prevalentes por orden de frecuencia fueron: candidiasis vaginal, tricomoniasis, condilomatosis, sífilis, gonorrea, vaginosis bacteriana y herpes.

El análisis comparado de cada variable (Tabla 1) mostró que el grupo con ETS vs. el control no tenían hijos, ni pareja estable o tenían mas de una pareja, tenían menos educación superior, un mayor porcentaje trabajaba fuera de Santiago y en la calle, y ejercían mas el comercio sexual. No existía un buen CSP estático y dinámico vs. el grupo control.

El modelo obtenido mediante análisis multivariado por regresión logística con las siguientes variables, predice la incidencia declarada de ETS SIDA en un 30\% de los casos ( $\mathrm{p}>$ chi2 0.00): CSP estático malo ( $\mathrm{OR}=1.34$; 95\% IC: 1.13-3.2), CSP dinámico malo $(\mathrm{OR}=4.80 ; 95 \%$ IC: $1.1-7.63)$, tienen hijos $(\mathrm{OR}=0.47 ; 95 \%$ IC: $-0.35-0.78)$, vivir fuera de Santiago (OR=1.27; 95\% IC: 1.11-4.3), trabajar en la calle $(\mathrm{OR}=3.92 ; 95 \% \mathrm{IC}: 1.19-7.4)$, ingreso bajo $(\mathrm{OR}=0.32 ; 95 \%$ IC: $-0.21-0.82)$, más de tres años de trabajo sexual $(\mathrm{OR}=0.32 ; 95 \% \mathrm{IC}$ : $0.02-1.19)$ y más de una pareja $(\mathrm{OR}=5.45 ; 95 \%$ IC: 0.98-10.5).

\section{DISCUSIÓN}

La ocurrencia de ETS encontrada en el grupo es de 3,5 casos por año. Al compararla con la población de referencia (mujeres embarazadas controladas), se observa una incidencia similar. La incidencia declarada de ETS por persona es menor ya que un $30 \%$ repite la infección en el año. Las patologías más prevalentes fueron
Tabla 1. Clima social preventivo y factores sociales en grupo con ETS vs. grupo control

\begin{tabular}{|c|c|c|c|}
\hline Características & \begin{tabular}{|l|} 
Grupo \\
ETS \\
$(\mathbf{n}=36)$
\end{tabular} & $\begin{array}{l}\text { Grupo } \\
\text { Control } \\
(n=84)\end{array}$ & $\begin{array}{l}\text { P Value } \\
(.05)\end{array}$ \\
\hline $\begin{array}{l}\text { Edad } \\
<25 \text { años }(\%) \\
>25 \text { años }(\%)\end{array}$ & $\begin{array}{r}8(23) \\
28(77)\end{array}$ & $\begin{array}{l}20(24.4) \\
64(75.5)\end{array}$ & \\
\hline $\begin{array}{l}\text { Pareja estable } \\
\text { Sin pareja }(\%) \\
\text { Con pareja }(\%)\end{array}$ & $\begin{array}{l}24(67) \\
12(33)\end{array}$ & $\begin{array}{l}54(65.1) \\
30(34.8)\end{array}$ & .049 \\
\hline $\begin{array}{l}\text { Número de hijos } \\
\text { Sin hijos }(\%) \\
\text { Con hijos }(\%)\end{array}$ & $\begin{array}{r}5(13) \\
31(87)\end{array}$ & $\begin{array}{r}9(9.3) \\
75(90.7)\end{array}$ & .05 \\
\hline $\begin{array}{l}\text { Escolaridad } \\
\text { Analfabetas }(\%) \\
\text { Educación básica }(\%) \\
\text { Educación media }(\%) \\
\text { Educación Superior }(\%)\end{array}$ & $\begin{array}{l}1(2.7) \\
9(26.1) \\
24(65) \\
2(6.1)\end{array}$ & $\begin{array}{r}1(2.3) \\
23(27.9) \\
54(61.6) \\
6(8.1) \\
\end{array}$ & .05 \\
\hline $\begin{array}{l}\text { Domicilio } \\
\text { En Santiago -urbano (\%) } \\
\text { Fuera Santiago -rural (\%) }\end{array}$ & $\begin{array}{r}30(86) \\
6(14)\end{array}$ & $\begin{array}{l}74(88) \\
10(11.6)\end{array}$ & $\begin{array}{l}.055 \\
.048\end{array}$ \\
\hline $\begin{array}{l}\text { Lugar de trabajo sexual } \\
\text { Clubes }(\%) \\
\text { Saunas y casas de masaje (\%) } \\
\text { Burdeles }(\%) \\
\text { Calle }(\%)\end{array}$ & $\begin{array}{r}31(85) \\
2(5.5) \\
1 \quad(3) \\
2(6.4)\end{array}$ & $\begin{array}{rr}72 & (86) \\
4 & (5.8) \\
2 & (3.4) \\
4 & (4.6)\end{array}$ & \\
\hline $\begin{array}{l}\text { Nivel de ingreso } \\
<\$ 500.000(\%) \\
>\$ 500.000(\%)\end{array}$ & $\begin{array}{r}30(86) \\
6(14)\end{array}$ & $\begin{array}{l}71(85) \\
13(15)\end{array}$ & \\
\hline $\begin{array}{l}\text { Edad de inicio sexual } \\
<16 \text { años }(\%) \\
>16 \text { años }(\%)\end{array}$ & $\begin{array}{l}11(33) \\
25(67)\end{array}$ & $\begin{array}{l}28(33.8) \\
56(66.2)\end{array}$ & \\
\hline $\begin{array}{l}\text { Tiempo de trabajo sexual } \\
<3 \text { años }(\%) \\
>3 \text { años }(\%)\end{array}$ & $\begin{array}{l}12(35) \\
24(65)\end{array}$ & $\begin{array}{l}28(34) \\
56(66)\end{array}$ & \\
\hline $\begin{array}{l}\text { Tipo de conducta sexual } \\
\text { Heterosexual }(\%) \\
\text { Homo y bisexual (\%) }\end{array}$ & $\begin{array}{r}33(94) \\
3(6)\end{array}$ & $\begin{array}{r}78(93) \\
6(7)\end{array}$ & \\
\hline $\begin{array}{l}\text { Número de parejas sexuales } \\
1(\%) \\
>1(\%)\end{array}$ & $\begin{array}{l}10(26) \\
26(74)\end{array}$ & $\begin{array}{l}28(32) \\
56(68)\end{array}$ & $\begin{array}{l}.05 \\
.04\end{array}$ \\
\hline $\begin{array}{l}\text { Comercio sexual } \\
\text { No }(\%) \\
\text { Sí }(\%)\end{array}$ & $\begin{array}{l}10(26) \\
26(74)\end{array}$ & $\begin{array}{l}28(32) \\
56(68)\end{array}$ & $\begin{array}{l}.05 \\
.04\end{array}$ \\
\hline $\begin{array}{l}\text { Uso de preservativos } \\
\text { Siempre }(\%) \\
\text { A veces o nunca }(\%)\end{array}$ & $\begin{array}{r}29(81) \\
7(19)\end{array}$ & $\begin{array}{l}67(80) \\
17(20)\end{array}$ & \\
\hline $\begin{array}{l}\text { Consumo alcohol o drogas } \\
\text { No }(\%) \\
\text { Sí }(\%)\end{array}$ & $\begin{array}{r}4(9) \\
32(91)\end{array}$ & $\begin{array}{l}10(11) \\
74(89)\end{array}$ & .05 \\
\hline $\begin{array}{l}\text { Buen CSP estático } \\
\text { Sí }(\%) \\
\text { No }(\%)\end{array}$ & $\begin{array}{r}3(6) \\
33(94)\end{array}$ & $\begin{array}{r}6(7) \\
78(93)\end{array}$ & .055 \\
\hline $\begin{array}{l}\text { Buen CSP dinámico } \\
\text { Sí }(\%) \\
\text { No }(\%)\end{array}$ & $\begin{array}{r}8(21) \\
28(79)\end{array}$ & $\begin{array}{l}22(26) \\
62(74)\end{array}$ & $\begin{array}{l}.048 \\
.041\end{array}$ \\
\hline
\end{tabular}


similares en la población de referencia. En los últimos años no se ha detectado casos VIH (+), probablemente porque el grupo de trabajadoras sexuales que asiste a control sanitario es muy seleccionado, pudiendo existir otros grupos con mayor incidencia que no consultan.

El análisis comparado entre el grupo de trabajadoras sexuales que no ha presentado ETS en el último año y la población en estudio encontró que ellas, en un mayor porcentaje, son menores de 25 años; tienen pareja estable e hijos; mayor escolaridad o más de 13 años de estudios y viven en el Santiago, factores que podrían ser protectores al influir sobre las conductas de riesgo. También se encontró en este grupo menos promiscuidad, expresada en un menor porcentaje de número de parejas sexuales y de mujeres que ejercen el comercio sexual, lo que concuerda con los datos de la literatura. Las demás variables estudiadas parecen comportarse en forma similar entre los grupos.

Sin embargo, en el análisis de regresión encontramos significancia estadística entre la incidencia declarada de ETS y el CSP estático y dinámico, es decir, aquellas trabajadoras sexuales con ETS no tenían buen CSP estático o percibían más riesgo de contagiarse, y también no tenían buen CSP dinámico, es decir, el grupo de pares recomendaba menos las conductas preventivas. En cambio, las mujeres con buen CSP tenían menos ETS, lo que podría estar reflejando un factor protector para la ocurrencia de ETS y podría servir para futuras estrategias de intervención.

En concordancia con el análisis simple de los datos se encontró asociación entre incidencia declarada de ETS y otros factores sociales.

Algunos de ellos fueron factores de riesgo como vivir fuera de Santiago en comunas rurales, trabajar en la calle, probablemente por un factor de acceso a la atención; y tener más de 1 pareja sexual. Otros, como tener hijos, mayor ingreso y más de 3 años como trabajadora sexual fueron factores sociales protectores.

Para algunos de los factores estudiados no se encontró asociación clara con la incidencia declarada de ETS. Es necesario realizar más estudios prospectivos para obtener mayores conclusiones sobre el efecto del Clima Social y los factores sociales estudiados en la incidencia de ETS.

En conclusión, el Clima Social Preventivo favorable y otros factores sociales influirían en las conductas de riesgo reduciendo la ocurrencia de ETS en el grupo estudiado. Esta información podría ser útil en Salud Pública al diseñar estrategias de prevención en este y otros grupos de riesgo.

\section{REFERENCIAS}

1. OMS. GINEBRA 1983. "Estrategia Mundial contra el SIDA". Serie OMS sobre SIDA, No11.

2. AGACFIDAN A., KAHL P. "Sexually Transmitted Diseases (STDs in the world)". FEMS Inmunol. Med. Microbiol. Jul 15 1999, 24(4): 431-5.

3. CARES W. JR. "Estimates of the incidence and prevalence of sexually transmitted diseases in the United States. American Social Health Association Panel. Sex. Transm. Dis. Apr. 1999, 26(4suppl): S2-7.

4. EBRAHIM S. H., PETERMAN T. A., ZAIDI A. A., KAMB M. L. "Mortality related to sexually transmitted diseases in U.S . women 1973 through 1992. Am. J. Public. Health. Jun 1997, 87(6): 938-44.

5. ANDREW N. "Sexually Transmitted Diseases: a review (see comments). J. Ark. Med. Soc. Nov. 1997,94(6): 251-4.

6. OPS. DIVISIÓN DE PREVENCIÓN Y CONTROL DE ENFERMEDADES, PROGRAMA REGIONAL DE SIDA /ETS. SIDA EN LAS AMÉRICAS. WASHINGTON D.C.: OPS, 1999. SIDA e Infecciones de Transmisión Sexual en las Américas. Rev. Panam. Salud Pública: 6(3); 1995: 215-9.

7. KROWCHU K.D.P. "Sexually Transmitted Diseases in Adolescents: an update. Compr. Ther. May 1999, 25(5): 265-71.

8. HUERTA K., BERKELHAMOR S., KLEIM J., AMMERMAN S., CHANG J., PROBER C.G., "Epidemiology of herpes simplex virus type 2 infections in Health". Jun 1996, 18 (6): 384-6.

9. JONSSON M., KARLSSON R., RYLANDER E. GUSTAVSON A., WADELL G. "The associactions between risk behavior and reported history of sexually transmitted diseases, among young women a population-based study". Int. J. STD. AIDS. Aug. 1997, 8 (8): 501-5.

10. HSER V.I., CHOU C.P., HOFFMAN V., ANGLIN M.D. "Cocaine use and high risk sexual behavior among STD clinic patients". Sex. Transm. Dis. Feb. 1999, 26 (2): 82-6.

11. BASEMAN J., ROSS M., WILLIAMS M. "Sale of sex for drugs and drugs for sex: an economic 
contextof sexual risk behavior for STDs". Sex. Transm. Dis. Sep. 1999, 26 (8): 444-9.

12. COMISIÓN NACIONAL DEL SIDA. Área de ETS. "Boletín Epidemiológico de ETS". Ministerio de Salud. Republican de Chile, 2000.

13. TAMAYA M., NAYKAYAMA H., SAKUMOTO M., MATSUMOTO T., AKAZAMA K., KUMAZAWA J. "Trends in sexually transmitted diseases and Condon use patterns among commercial sex workers in Fukuoka City, Japan 1990-93. Genitourin. Med. Oct. 1996, 72 (5): 358-61.

14. WEIR S.S., FELDBLEIM P.J. "Condom use to prevent incident STDs (letter comment)". Sex. Trasn. Dis. Jan.-Feb.1996, 23 (1):76-7; discussion 79-82.

15. FONTANET A.C., SABA J., CHANDELYING V., SAKONDHAVAT C., BRIRALEUS P., RUGPAO S., WOGSOMCHAI C., KIRIWAT O., TAWABUTRA S., DAPLY L., LANGE S.M., ROJANAPITHAYAKORN. "Protection against sexually transmitted diseases by granting sex workers in Thailand the choice of using the male or female condom results from a randomized controlled trial". AIDS Oct. 1998, 12 (14): 1851-9.

16. WONG M.L., CHAN R.K., CHUA W.L., WEE S "Sexually transmitted diseases and condom use among female freelance and brothel based sex workers in Singapore". Sex Transm. Dis. Nov. 1999, 26 (10): 593-600.

17. TRUJILLO L., MUÑOZ D., GOTUZZO E., VI A., WATTS D.M. "Sexual practices and prevalence of HIV, HTLV-I II, and Treponema pallidium among clandestine female sex workers in Lima, Perú. Sex. Transm. Dis. Feb. 1999, 26 (2): 115-8.

18. BERMAN S.M., GUNN R.A., ARAL S.D. "Abstinence and safer sex among adolescents (letter). JAMA. Apr. 28, 1999, 281 (16): 1485; discussion 1487-8.

19. OMS. "Guidelines on Sexually Transmitted Diseases and AIDS for Family Planning Programs ".1989.

20. OMS. La Educación Sanitaria Escolar en la Prevención del SIDA y de las ETS. Serie OMS sobre SIDA, Nº 10, Ginebra 1992.
21. HERNÁNDEZ-GIRON C.A., CRUZ-VALDEZ A., FIGUEROS L.J., HERNÁNDEZ-AVILA M. "Prevalence and risk factors associated with syphilis in women". Rev. Salud Pública. Dec. 1998, 32 (6): 579-86.

22. RUGPAO S., WANAPIRAK C., SIRIGRATIYAKUL S., YUTABOOTR Y., PROSETWITAYAKIJ W., SUWARKITI S., WONGWORAAPT K., TAVANABUTRA S., NATPROTAN C., SABA J., "Sexually transmitted diseases prevalence in brothel-based commercial sex workers in Chiang Min Thailand impact of the condom use campaign". J. Med.Assoc. Thai. Jul. 1997, 80 (7): 426-30.

23. SHAIN R.N., PIPER J.M., NEWTON E.R., PERDUE S.T., RAMOS R., CHAMPION J.D, GUERRA F.A. A randomized, controlled trial of a behavioral intervention to prevent sexually transmitted diseases among minority women". N. Engl. J. Med. Jan 14, 1999, 340 (2): 93-100.

24. TIMASHEFF N.S. Durkeim en Sociological Theory, Its nature and growth. Random House N. Y., 1967: 109-11.

25. PEREDA C., L. CARIS L. (1999') "Redes sociales y clima de prevención del consumo de $\mathrm{OH}$ y drogas en la Comuna de Los Vilos". Depto de Salud Mental. Escuela de Salud Pública Santiago, Chile.

26. PEREDA C., L. CARIS L., BUSTAMANTE J.(1999b) "Redes de amigos y clima de prevención del consumo de $\mathrm{OH}$ y drogas de la Comuna de Los Vilos". Depto de Salud Mental. Escuela de Salud Pública Santiago, Chile.

27. CONACE "Estudio Nacional sobre el consumo de alcohol, tabaco y drogas y el clima social preventivo de la población escolar de Chile". Informe Ejecutivo. 2000.

28. HERNÁNDEZ S. "Clima Social Preventivo de ETS SIDA y Características Socio Demográfico Conductuales de Las Trabajadoras Sexuales en Control Sanitario en El Consultorio $N^{\circ} 1$ ". Tesis de Grado para Magister en Salud Pública Mención Epidemiología. Año 2000. Escuela Salud Pública. Universidad de Chile. 